\title{
Resuming Anticoagulation following Upper Gastrointestinal Bleeding among Patients with Nonvalvular Atrial Fibrillation-A Microsimulation Analysis
}

\author{
Matthew A Pappas, MD, MPH',2*; Natalie Evans, MD³; Maged K Rizk, MD, MBA; Michael B Rothberg, MD, MPH
}

\begin{abstract}
${ }^{1}$ Cleveland Clinic, Medicine Institute, Center for Value-based Care Research, Cleveland, Ohio; ${ }^{2}$ Cleveland Clinic, Medicine Institute, Department of Hospital Medicine, Cleveland, Ohio; ${ }^{3}$ Cleveland Clinic, Heart and Vascular Institute, Department of Vascular Medicine, Cleveland, Ohio; ${ }^{4} \mathrm{Cleveland}$ Clinic, Digestive Disease and Surgery Institute, Department of Gastroenterology, Cleveland, Ohio.
\end{abstract}

BACKGROUND: Among patients with nonvalvular atrial fibrillation (NVAF) who have sustained an upper gastrointestinal bleed (UGIB), the benefits and harms of oral anticoagulation change over time. Early resumption of anticoagulation increases recurrent bleeding, while delayed resumption exposes patients to a higher risk of ischemic stroke. We therefore set out to estimate the expected benefit of resuming anticoagulation as a function of time after UGIB among patients with NVAF.

METHODS: We created a decision-analytic model estimating discounted quality-adjusted life-years when patients with NVAF resume anticoagulation on each day following UGIB. We simulated from a health system perspective over a lifelong time horizon.

RESULTS: Peak utility for warfarin was achieved by resumption 41 days after hemostasis from the index UGIB. Resumption between days 32 and 51 produced greater than $99.9 \%$ of the peak utility. Peak utility for apixaban was achieved by resumption 32 days after the index UGIB. Resumption between days 21 and 47 produced greater than $99.9 \%$ of the peak utility. Of input parameters, results were most sensitive to underlying stroke risk. Specifically, across the range of $\mathrm{CHA}_{2} \mathrm{DS}_{2}$-Vasc scores, the optimal day of resumption varied by around 11 days for patients resuming warfarin and by around 15 days for patients resuming apixaban. Results were less sensitive to underlying risk of rebleeding.

CONCLUSIONS: For patients with NVAF following UGIB, warfarin is optimally restarted approximately six weeks following hemostasis, and apixaban is optimally restarted approximately one month following hemostasis. Modest changes to this timing based on probability of thromboembolic stroke are reasonable. Journal of Hospital Medicine 2019;14:394-400. Published online first April 8, 2019. () 2019 Society of Hospital Medicine

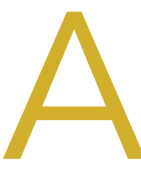

nticoagulation is commonly used in the management of atrial fibrillation to reduce the risk of ischemic stroke. Warfarin and other anticoagulants increase the risk of hemorrhagic complications, including upper gastrointestinal bleeding (UGIB). Following $\mathrm{UGIB}$, management of anticoagulation is highly variable. Many patients permanently discontinue anticoagulation, while others continue without interruption..$^{1-4}$ Among patients who resume warfarin, different cohorts have measured median times to resumption ranging from four days to 50 days..$^{1-3}$ Outcomes data are sparse, and clinical guidelines offer little direction. ${ }^{5}$

Following UGIB, the balance between the risks and benefits of anticoagulation changes over time. Rebleeding risk is highest immediately after the event and declines quickly; therefore, rapid resumption of anticoagulation causes patient harm. ${ }^{3}$ Meanwhile, the risk of stroke remains constant, and delay in

\footnotetext{
*Corresponding Author: Matthew A Pappas, MD; E-mail: pappasm@ccf.org; Telephone: 216-444-9565

Additional Supporting Information may be found in the online version of this article.
}

Received: November 7, 2018; Revised: January 22, 2019;

Accepted: February 6, 2019

๑ 2019 Society of Hospital Medicine DOI 10.12788/jhm.3189 resumption of anticoagulation is associated with increased risk of stroke and death. ${ }^{1}$ At some point in time following the initial UGIB, the expected harm from bleeding would equal the expected harm from stroke. This time point would represent the optimal time to restart anticoagulation.

Trial data are unlikely to identify the optimal time for restarting anticoagulation. A randomized trial comparing discrete reinitiation times (eg, two weeks vs six weeks) may easily miss the optimal timing. Moreover, because the daily probability of thromboembolic events is low, large numbers of patients would be required to power such a study. In addition, a number of oral anticoagulants are now approved for prevention of thromboembolic stroke in atrial fibrillation, and each drug may have different optimal timing.

In contrast to randomized trials that would be impracticable for addressing this clinical issue, microsimulation modeling can provide granular information regarding the optimal time to restart anticoagulation. Herein, we set out to estimate the expected benefit of reinitiation of warfarin, the most commonly used oral anticoagulant, ${ }^{6}$ or apixaban, the direct oral anticoagulant with the most favorable risk profile, as a function of days after UGIB.

\section{METHODS}

We previously described a microsimulation model of anticoagulation among patients with nonvalvular atrial fibrillation 
(NVAF; hereafter, we refer to this model as the Personalized Anticoagulation Decision-Making Assistance model, or PADMA) ${ }^{8,9}$ For this study, we extended this model to incorporate the probability of rebleeding following UGIB and include apixaban as an alternative to warfarin. This model begins with a synthetic population following UGIB, the members of which are at varying risk for thromboembolism, recurrent UGIB, and other hemorrhages. For each patient, the model simulates a number of possible events (eg, thromboembolic stroke, intracranial hemorrhage, rebleeding, and other major extracranial hemorrhages) on each day of an acute period of 90 days after hemostasis. Patients who survive until the end of the acute period enter a simulation with annual, rather than daily, cycles. Our model then estimates total quality-adjusted life-years (QALYs) for each patient, discounted to the present. We report the average discounted QALYs produced by the model for the same population if all individuals in our input population were to resume either warfarin or apixaban on a specific day. Input parameters and ranges are summarized in Table 1, a simplified schematic of our model is shown in the Supplemental Appendix, and additional details regarding model structure and assumptions can be found in earlier work. ${ }^{8,9}$ We simulated from a health system perspective over a lifelong time horizon. All analyses were performed in version 14 of Stata (StataCorp, LLC, College Station, Texas).

\section{Synthetic Population}

To generate a population reflective of the comorbidities and age distribution of the US population with NVAF, we merged relevant variables from the National Health and Nutrition Examination Survey (NHANES; 2011-2012), using multiple imputation to correct for missing variables. ${ }^{10}$ We then bootstrapped to national population estimates by age and sex to arrive at a hypothetical population of the United States. ${ }^{11}$ Because NHANES does not include atrial fibrillation, we applied sexand age-specific prevalence rates from the AnTicoagulation and Risk Factors In Atrial Fibrillation study.12 We then calculated commonly used risk scores $\left(\mathrm{CHA}_{2} \mathrm{DS}_{2}\right.$-Vasc and HAS-BLED) for each patient and limited the population to patients with a $\mathrm{CHA}_{2} \mathrm{DS}_{2}$-Vasc score of one or greater. ${ }^{13,14}$ The population resuming apixaban was further limited to patients whose creatinine clearance was $25 \mathrm{~mL} / \mathrm{min}$ or greater in keeping with the entry criteria in the phase 3 clinical trial on which the medication's approval was based. ${ }^{15}$

To estimate patient-specific probability of rebleeding, we generated a Rockall score for each patient. ${ }^{16}$ Although the discrimination of the Rockall score is limited for individual patients, as with all other tools used to predict rebleeding following UGIB, the Rockall score has demonstrated reasonable calibration across a threefold risk gradient. ${ }^{17-19}$ International consensus guidelines recommend the Rockall score as one of two risk prediction tools for clinical use in the management of patients with UGIB. ${ }^{20}$ In addition, because the Rockall score includes some demographic components (five of a possible 11 points), our estimates of rebleeding risk are covariant with other patient-specific risks. We assumed that the endoscopic components of the Rockall score were present in our cohort at the same frequency as in the original derivation and are independent of known patient risk factors. ${ }^{16}$ For example, 441 out of 4,025 patients in the original Rockall derivation cohort presented with a systolic blood pressure less than $100 \mathrm{~mm} \mathrm{Hg}$. We assumed that an independent and random 10.96\% of the cohort would present with shock, which confers two points in the Rockall score.

The population was replicated 60 times, with identical copies of the population resuming anticoagulation on each of days 1-60 (where day zero represents hemostasis). Intermediate data regarding our simulated population can be found in the Supplemental Appendix and in prior work.

\section{Event Type, Severity, and Mortality}

Each patient in our simulation could sustain several discrete and independent events: ischemic stroke, intracranial hemorrhage, recurrent UGIB, or extracranial major hemorrhage other than recurrent UGIB. As in prior analyses using the PADMA model, we did not consider minor hemorrhagic events. ${ }^{8}$

The probability of each event was conditional on the corresponding risk scoring system. Patient-specific probability of ischemic stroke was conditional on $\mathrm{CHA}_{2} \mathrm{DS}_{2}$-Vasc score..$^{21,22}$ Patient-specific probability of intracranial hemorrhage was conditional on HAS-BLED score, with the proportions of intracranial hemorrhage of each considered subtype (intracerebral, subarachnoid, or subdural) bootstrapped from previously-published data. ${ }^{21-24}$ Patient-specific probability of rebleeding was conditional on Rockall score from the combined Rockall and Vreeburg validation cohorts. ${ }^{17}$ Patient-specific probability of extracranial major hemorrhage was conditional on HAS-BLED score. ${ }^{21}$ To avoid double-counting of UGIB, we subtracted the baseline risk of UGIB from the overall rate of extracranial major hemorrhages using previously-published data regarding relative frequency and a bootstrapping approach. ${ }^{25}$

\section{Probability of Rebleeding Over Time}

To estimate the decrease in rebleeding risk over time, we searched the Medline database for systematic reviews of recurrent bleeding following UGIB using the strategy detailed in the Supplemental Appendix. Using the interval rates of rebleeding we identified, we calculated implied daily rates of rebleeding at the midpoint of each interval. For example, 39.5\% of rebleeding events occurred within three days of hemostasis, implying a daily rate of approximately $13.2 \%$ on day two (32 of 81 events over a three-day period). We repeated this process to estimate daily rates at the midpoint of each reported time interval and fitted an exponential decay function. ${ }^{26}$ Our exponential fitted these datapoints quite well, but we lacked sufficient data to test other survival functions (eg, Gompertz, lognormal, etc.). Our fitted exponential can be expressed as:

$$
P_{\text {rebleeding }}=b_{0}{ }^{*} \exp \left(b_{1}{ }^{*} \text { day }\right)
$$

where $b_{0}=0.1843$ (SE: 0.0136) and $b_{1}=-0.1563$ (SE: 0.0188). For example, a mean of $3.9 \%$ of rebleeding episodes will occur on day $10(0.1843 * \exp (-0.1563 * 10))$. 
TABLE 1. Summary of Model Input Parameters

\section{Fixed and Sampled Inputs}

Input parameter

Base-case estimate

References

Age and sex of US population

US Census

11

Age- and sex-specific prevalence of atrial fibrillation

ATRIA

12

Age- and sex-specific prevalence and covariation of stroke risk factors

NHANES

Annual incidence of ischemic stroke

Fixed for each $\mathrm{CHA}_{2} \mathrm{DS}_{2}$-Vasc score $(0.2 \%$ to $14.4 \%)$

Annual incidence of intracranial hemorrhage

Fixed for each HAS-BLED score (0.1\%-1.3\%)

Annual incidence of extracranial major hemorrhage

Fixed for each HAS-BLED score (0.5\% to $14.5 \%)$

Incidence of rebleeding

Fixed for each Rockall score based on combined rates

in Rockall and Vreeburg cohorts

Timing of rebleeding

Exponential decay model (see methods)

Subtypes of intracranial hemorrhage

$65 \%$ intracerebral, $6 \%$ subarachnoid, $29 \%$ subdural

Trajectories of INR after reinitiation of warfarin

Sampled from clinical warfarin initiation group of COAG trial

Inpatient mortality following ischemic or hemorrhagic stroke

Predicted

Inpatient mortality following subdural hemorrhage

Length of stay, conditioned on diagnosis

Predicted

Sampled

Hazard ratio for long-term mortality following stroke or $\mathrm{ICH}, \mathrm{mRS} \leq 2$

1.7

Hazard ratio for long-term mortality following stroke or $\mathrm{ICH}, \mathrm{mRS}=3$ or 4

2.9

2.9

8.3

Hazard ratio for long-term mortality following stroke or ICH, mRS 5

Varies

Baseline probability of death by age

Predicted using NINDS trial data

10

21,22

21,22

21,22

Future mRS following ischemic stroke

$13.8 \%$ each mRS $0-2,19.5 \%$ each mRS 3-5

Future mRS following $\mathrm{ICH}$, conditional on survival to discharge

0.046

Disutility, mRS 1

0.212

Disutility, mRS 2

0.331

Disutility, mRS 3

0.331

Disutility, mRS 4

0.652

Disutility, mRS 5

0.944

\section{Continuously Varying Input Parameters}

\begin{tabular}{|c|c|c|c|c|}
\hline Input Parameter & Mean (Median) & $\mathrm{sd}(\mathrm{IQR})$ & Distribution & References \\
\hline Incidence of rebleeding & $17.8 \%$ & $0.8 \%$ & Normal & 17 \\
\hline Percentage of major extracranial hemorrhagic events that are gastrointestinal & $35.2 \%$ & $2.2 \%$ & Normal & 25 \\
\hline Percentage of $\mathrm{GI}$ bleeds that arise from the upper GI tract & $75.0 \%$ & $5.1 \%$ & Normal & 25 \\
\hline Severity of ischemic strokes (NIHSS) & 16.2 & 7.0 & Normal & 38,39 \\
\hline Severity of intracerebral hemorrhages (NIHSS) & 9 & $(3-19)$ & Normal & 39 \\
\hline Severity of subarachnoid hemorrhages (NIHSS) & 3 & $(0-11)$ & Gamma & 39 \\
\hline In-hospital mortality following extracranial major hemorrhage & $9.5 \%$ & $3.4 \%$ & Normal & 29,43 \\
\hline Discount rate & $3 \%$ & $1.7 \%$ & Uniform $(0 \%-6 \%)$ & 44 \\
\hline
\end{tabular}

Abbreviations: GI, gastrointestinal; ICH, intracerebral hemmorhage; mRS, modified Rankin Score; NIHSS, NIH Stroke Scale. 
TABLE 2. Optimal Day of Warfarin or Apixaban Reinitiation by $\mathrm{CHA}_{2} \mathrm{DS}_{2}$-Vasc Score ${ }^{\mathrm{a}}$

\begin{tabular}{lcc}
\hline $\mathrm{CHA}_{2} \mathrm{DS}_{2}$-Vasc & Apixaban & Warfarin \\
\hline 1 & $52(49-55)$ & $50(48-52)$ \\
\hline 2 & $49(46-52)$ & $48(46-50)$ \\
\hline 3 & $46(43-49)$ & $46(44-47)$ \\
\hline 4 & $43(40-46)$ & $43(42-45)$ \\
\hline 6 & $40(37-43)$ & $41(39-43)$ \\
\hline 7 & $37(34-40)$ & $39(37-40)$ \\
\hline 9 & $34(31-37)$ & $36(35-38)$ \\
\hline
\end{tabular}

${ }^{a}$ Day 0 represents hemostasis from the index UGIB. The range of predicted days on which initiation would confer at least $99.99 \%$ of the peak QALYs is shown in parentheses

\section{Relative Risks of Events with Anticoagulation}

For patients resuming warfarin, the probabilities of each event were adjusted based on patient-specific daily INR. All INRs were assumed to be 1.0 until the day of warfarin reinitiation, after which interpolated trajectories of postinitiation INR measurements were sampled for each patient from an earlier study of clinical warfarin initiation. ${ }^{27}$ Relative risks of ischemic stroke and hemorrhagic events were calculated based on each day's INR.

For patients taking apixaban, we assumed that the medication would reach full therapeutic effect one day after reinitiation. Based on available evidence, we applied the relative risks of each event with apixaban compared with warfarin. ${ }^{25}$

\section{Future Disability and Mortality}

Each event in our simulation resulted in hospitalization. Length of stay was sampled for each diagnosis. ${ }^{28}$ The disutility of hospitalization was estimated based on length of stay. ${ }^{8}$ Inpatient mortality and future disability were predicted for each event as previously described. ${ }^{8}$ We assumed that recurrent episodes of UGIB conferred morbidity and mortality identical to extracranial major hemorrhages more broadly. 29,30

\section{Disutilities}

We used a multiplicative model for disutility with baseline utilities conditional on age and sex. ${ }^{31}$ Each day after resumption of anticoagulation carried a disutility of 0.012 for warfarin or 0.002 for apixaban, which we assumed to be equivalent to aspirin in disutility. ${ }^{32}$ Long-term disutility and life expectancy were conditional on modified Rankin Score (mRS). ${ }^{33,34}$ We discounted all QALYs to day zero using standard exponential discounting and a discount rate centered at $3 \%$. We then computed the average discounted QALYs among the cohort of patients that resumed anticoagulation on each day following the index UGIB.

\section{Sensitivity Analyses and Metamodel}

To assess sensitivity to continuously varying input parameters, such as discount rate, the proportion of extracranial major hemorrhages that are upper Gl bleeds, and inpatient mortality from extracranial major hemorrhage, we constructed a metamodel (a regression model of our microsimulation results). ${ }^{35}$ We tested for interactions among input parameters and dropped parameters that were not statistically significant predictors of discounted QALYs from our metamodel. We then tested for interactions between each parameter and day resuming anticoagulation to determine which factors may impact the optimal day of reinitiation. Finally, we used predicted marginal effects from our metamodel to assess the change in optimal day across the ranges of each input parameter when other parameters were held at their medians.

\section{RESULTS}

Resuming warfarin on day zero produced the fewest QALYs. With delay in reinitiation of anticoagulation, expected QALYs increased, peaked, and then declined for all scenarios. In our base-case simulation of warfarin, peak utility was achieved by resumption 41 days after the index UGIB. Resumption between days 32 and 51 produced greater than $99.9 \%$ of peak utility. In our base-case simulation of apixaban, peak utility was achieved by resumption 32 days after the index UGIB. Resumption between days 21 and 47 produced greater than $99.9 \%$ of peak utility. Results for warfarin and apixaban are shown in Figures 1 and 2, respectively.

The optimal day of warfarin reinitiation was most sensitive to $\mathrm{CHA}_{2} \mathrm{DS}_{2}$-Vasc scores and varied by around 11 days between a $\mathrm{CHA}_{2} \mathrm{DS}_{2}$-Vasc score of one and a $\mathrm{CHA}_{2} \mathrm{DS}_{2}$-Vasc score of six (the $5^{\text {th }}$ and $95^{\text {th }}$ percentiles, respectively) when all other parameters are held at their medians. Results were comparatively insensitive to rebleeding risk. Varying Rockall score from two to seven (the $5^{\text {th }}$ and $95^{\text {th }}$ percentiles, respectively) added three days to optimal warfarin resumption. Varying other parameters from the $5^{\text {th }}$ to the $95^{\text {th }}$ percentile (including HAS-BLED score, sex, age, and discount rate) changed expected QALYs but did not change the optimal day of reinitiation of warfarin. Optimal day of reinitiation for warfarin stratified by $\mathrm{CHA}_{2} \mathrm{DS}_{2}$-Vasc score is shown in Table 2.

Sensitivity analyses for apixaban produced broadly similar results, but with greater sensitivity to rebleeding risk. Optimal day of reinitiation varied by 15 days over the examined range of $\mathrm{CHA}_{2} \mathrm{DS}_{2}$-Vasc scores (Table 2) and by six days over the range of Rockall scores (Supplemental Appendix). Other input parameters, including HAS-BLED score, age, sex, and discount rate, changed expected QALYs and were significant in our metamodel but did not affect the optimal day of reinitiation. Metamodel results for both warfarin and apixaban are included in the Supplemental Appendix.

\section{DISCUSSION}

Anticoagulation is frequently prescribed for patients with NVAF, and hemorrhagic complications are common. Although anticoagulants are withheld following hemorrhages, scant evidence to inform the optimal timing of reinitiation is available. 


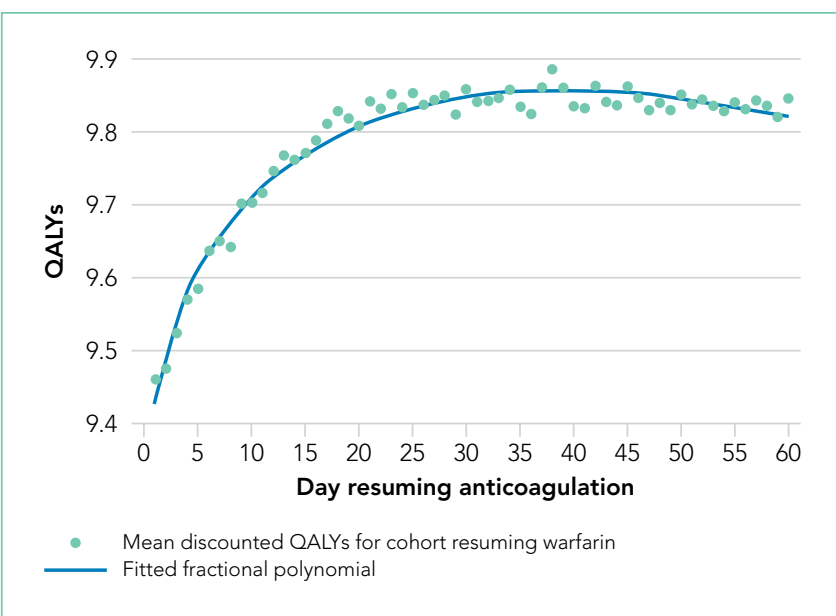

FIG 1. Expected Average QALYS Conferred as a Function of Day on which Warfarin is Resumed. Day 0 represents hemostasis of the index UGIB. The fitted line is a fractional polynomial.

Abbreviations: QALYs, quality-adjusted life-years; UGIB; upper gastrointestinal bleeding

In this microsimulation analysis, we found that the optimal time to reinitiate anticoagulation following UGIB is around 41 days for warfarin and around 32 days for apixaban. We have further demonstrated that the optimal timing of reinitiation can vary by nearly two weeks, depending on a patient's underlying risk of stroke, and that early reinitiation is more sensitive to rebleeding risk than late reinitiation.

Prior work has shown that early reinitiation of anticoagulation leads to higher rates of recurrent hemorrhage while failure to reinitiate anticoagulation is associated with higher rates of stroke and mortality. ${ }^{1-4,36}$ Our results add to the literature in a number of important ways. First, our model not only confirms that anticoagulation should be restarted but also suggests when this action should be taken. The competing risks of bleeding and stroke have left clinicians with little guidance; we have quantified the clinical reasoning required for the decision to resume anticoagulation. Second, by including the disutility of hospitalization and long-term disability, our model more accurately represents the complex tradeoffs between recurrent hemorrhage and (potentially disabling) stroke than would a comparison of event rates. Third, our model is conditional upon patient risk factors, allowing clinicians to personalize the timing of anticoagulation resumption. Theory would suggest that patients at higher risk of ischemic stroke benefit from earlier resumption of anticoagulation, while patients at higher risk of hemorrhage benefit from delayed reinitiation. We have quantified the extent to which patient-specific risks should change timing. Fourth, we offer a means of improving expected health outcomes that requires little more than appropriate scheduling. Current practice regarding resuming anticoagulation is widely variable. Many patients never resume warfarin, and those that do resume do so after highly varied periods of time. ${ }^{1-5,36}$ We offer a means of standardizing clinical practice and improving expected patient outcomes.

Interestingly, patient-specific risk of rebleeding had little effect on our primary outcome for warfarin, and a greater effect

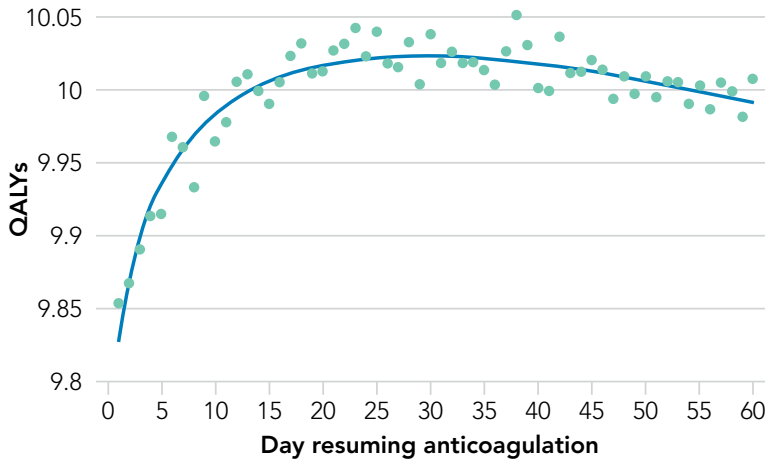

- Mean discounted QALYs for cohort resuming warfarin Fitted fractional polynomial

FIG 2. Expected Average QALYs Conferred as a Function of Day on which Apixaban is Resumed. Day 0 represents hemostasis of the index UGIB. The fitted line is a fractional polynomial.

Abbreviations: QALYs, quality-adjusted life-years; UGIB; upper gastrointestinal bleeding

in our simulation of apixaban. It would seem that rebleeding risk, which decreases roughly exponentially, is sufficiently low by the time period at which warfarin should be resumed that patient-specific hemorrhage risk factors have little impact. Meanwhile, at the shorter post-event intervals at which apixaban can be resumed, both stroke risk and patient-specific bleeding risk are worthy considerations.

Our model is subject to several important limitations. First, our predictions of the optimal day as a function of risk scores can only be as well-calibrated as the input scoring systems. It is intuitive that patients with higher risk of rebleeding benefit from delayed reinitiation, while patients with higher risk of thromboembolic stroke benefit from earlier reinitiation. Still, clinicians seeking to operationalize competing risks through these two scores-or, indeed, any score-should be mindful of their limited calibration and shared variance. In other words, while the optimal day of reinitiation is likely in the range we have predicted and varies to the degree demonstrated here, the optimal day we have predicted for each score is likely overly precise. However, while better-calibrated prediction models would improve the accuracy of our model, we believe ours to be the best estimate of timing given available data and this approach to be the most appropriate way to personalize anticoagulation resumption.

Our simulation of apixaban carries an additional source of potential miscalibration. In the clinical trials that led to their approval, apixaban and other direct oral anticoagulants (DOACs) were compared with warfarin over longer periods of time than the acute period simulated in this work. Over a short period of time, patients treated with more rapidly therapeutic medications (in this case, apixaban) would receive more days of effective therapy compared with a slower-onset medication, such as warfarin. Therefore, the relative risks experienced by patients are likely different over the time period we have simulated compared with those measured over longer periods of time (as in phase 3 clinical trials). Our results for apixaban 
should be viewed as more limited than our estimates for warfarin. More broadly, simulation analyses are intended to predict overall outcomes that are difficult to measure. While other frameworks to assess model credibility exist, the fact remains that no extant datasets can directly validate our predictions. ${ }^{37}$

Our findings are limited to patients with NVAF. Anticoagulants are prescribed for a variety of indications with widely varied underlying risks and benefits. Models constructed for these conditions would likely produce different timing for resumption of anticoagulation. Unfortunately, large scale cohort studies to inform such models are lacking. Similarly, we simulated UGIB, and our results should not be generalized to populations with other types of bleeding (eg, intracranial hemorrhage). Again, cohort studies of other types of bleeding would be necessary to understand the risks of anticoagulation over time in such populations.

Higher-quality data regarding risk of rebleeding over time would improve our estimates. Our literature search identified only one systematic review that could be used to estimate the risk of recurrent UGIB over time. These data are not adequate to interrogate other forms this survival curve could take, such as Gompertz or Weibull distributions. Recurrence risk almost certainly declines over time, but how quickly it declines carries additional uncertainty.

Despite these limitations, we believe our results to be the best estimates to date of the optimal time of anticoagulation reinitiation following UGIB. Our findings could help inform clinical practice guidelines and reduce variation in care where current practice guidelines are largely silent. Given the potential ease of implementing scheduling changes, our results represent an opportunity to improve patient outcomes with little resource investment.

In conclusion, after UGIB associated with anticoagulation, our model suggests that warfarin is optimally restarted approximately six weeks following hemostasis and that apixaban is optimally restarted approximately one month following hemostasis. Modest changes to this timing based on probability of thromboembolic stroke are reasonable.

Disclosures: The authors have nothing to disclose.

Funding: The authors received no specific funding for this work.

\section{References}

1. Witt DM, Delate T, Garcia DA, et al. Risk of thromboembolism, recurrent hemorrhage, and death after warfarin therapy interruption for gastrointestinal tract bleeding. Arch Intern Med. 2012;172(19):1484-1491. doi: 10.1001/ archinternmed.2012.4261.

2. Sengupta N, Feuerstein JD, Patwardhan VR, et al. The risks of thromboembolism vs recurrent gastrointestinal bleeding after interruption of systemic anticoagulation in hospitalized inpatients with gastrointestinal bleeding: a prospective study. Am J Gastroenterol. 2015;110(2):328-335. doi: 10.1038/ ajg.2014.398

3. Qureshi W, Mittal C, Patsias I, et al. Restarting anticoagulation and outcomes after major gastrointestinal bleeding in atrial fibrillation. Am J Cardiol. 2014;113(4):662-668. doi: 10.1016/j.amjcard.2013.10.044

4. Milling TJ, Spyropoulos AC. Re-initiation of dabigatran and direct factor Xa antagonists after a major bleed. Am J Emerg Med. 2016;34(11):19-25. doi: 10.1016/j.ajem.2016.09.049
5. Brotman DJ, Jaffer AK. Resuming anticoagulation in the first week following gastrointestinal tract hemorrhage. Arch Intern Med. 2012;172(19):1492-1493. doi: 10.1001/archinternmed.2012.4309

6. Barnes GD, Lucas E, Alexander GC, Goldberger ZD. National trends in ambulatory oral anticoagulant use. Am J Med. 2015;128(12):1300-5. doi: 10.1016/j.amjmed.2015.05.044

7. Noseworthy PA, Yao X, Abraham NS, Sangaralingham LR, McBane RD, Shah ND. Direct comparison of dabigatran, rivaroxaban, and apixaban for effectiveness and safety in nonvalvular atrial fibrillation. Chest. 2016;150(6):13021312. doi: 10.1016/j.chest.2016.07.013.

8. Pappas MA, Barnes GD, Vijan S. Personalizing bridging anticoagulation in patients with nonvalvular atrial fibrillation-a microsimulation analysis. J Gen Intern Med. 2017;32(4):464-470. doi: 10.1007/s11606-016-3932-7.

9. Pappas MA, Vijan S, Rothberg MB, Singer DE. Reducing age bias in decision analyses of anticoagulation for patients with nonvalvular atrial fibrillation - a microsimulation study. PloS One. 2018;13(7):e0199593. doi: 10.1371/journal. pone.0199593.

10. National Center for Health Statistics. National Health and Nutrition Examination Survey. https://www.cdc.gov/nchs/nhanes/about_nhanes.htm. Accessed August 30, 2018

11. United States Census Bureau. Age and sex composition in the United States: 2014. https://www.census.gov/data/tables/2014/demo/age-and-sex/2014age-sex-composition.html. Accessed August 30, 2018

12. Go AS, Hylek EM, Phillips KA, et al. Prevalence of diagnosed atrial fibrillation in adults: national implications for rhythm management and stroke prevention: the AnTicoagulation and Risk Factors in Atrial Fibrillation (ATRIA) study. JAMA. 2001;285(18):2370-2375. doi: 10.1001/jama.285.18.2370.

13. Lip GYH, Nieuwlaat R, Pisters R, Lane DA, Crijns HJGM. Refining clinical risk stratification for predicting stroke and thromboembolism in atrial fibrillation using a novel risk factor-based approach: the euro heart survey on atrial fibrillation. Chest. 2010;137(2):263-272. doi: 10.1378/chest.09-1584.

14. Pisters R, Lane DA, Nieuwlaat R, de Vos CB, Crijns HJGM, Lip GYH. A novel user-friendly score (HAS-BLED) to assess 1-year risk of major bleeding in patients with atrial fibrillation. Chest. 2010;138(5):1093-1100. doi: 10.1378/ chest.10-0134.

15. Granger CB, Alexander JH, McMurray JJV, et al. Apixaban versus warfarin in patients with atrial fibrillation. N Engl J Med. 2011;365(11):981-992. doi: 10.1056/NEJMoa1107039.

16. Rockall TA, Logan RF, Devlin HB, Northfield TC. Risk assessment after acute upper gastrointestinal haemorrhage. Gut. 1996;38(3):316-321. doi: 10.1136/ gut.38.3.316.

17. Vreeburg EM, Terwee CB, Snel P, et al. Validation of the Rockall risk scoring system in upper gastrointestinal bleeding. Gut. 1999;44(3):331-335. doi: 10.1136/gut.44.3.331.

18. Enns RA, Gagnon YM, Barkun AN, et al. Validation of the Rockall scoring system for outcomes from non-variceal upper gastrointestinal bleeding in a Canadian setting. World J Gastroenterol. 2006;12(48):7779-7785. doi: 10.3748/ wjg.v12.i48.7779.

19. Stanley AJ, Laine L, Dalton HR, et al. Comparison of risk scoring systems for patients presenting with upper gastrointestinal bleeding: international multicentre prospective study. BMJ. 2017;356:i6432. doi: 10.1136/bmj.i6432.

20. Barkun AN, Bardou M, Kuipers EJ, et al. International consensus recommendations on the management of patients with nonvariceal upper gastrointestinal bleeding. Ann Intern Med. 2010;152(2):101-113. doi: 10.7326/0003-4819152-2-201001190-00009

21. Friberg $L$, Rosenqvist $M$, Lip GYH. Evaluation of risk stratification schemes for ischaemic stroke and bleeding in 182678 patients with atrial fibrillation: the Swedish atrial fibrillation cohort study. Eur Heart J. 2012;33(12):1500-1510. doi: 10.1093/eurheartj/ehr488.

22. Friberg L, Rosenqvist M, Lip GYH. Net clinical benefit of warfarin in patients with atrial fibrillation: a report from the Swedish atrial fibrillation cohort study. Circulation. 2012;125(19):2298-2307. doi: 10.1161/CIRCULATIONAHA.111.055079.

23. Hart RG, Diener HC, Yang S, et al. Intracranial hemorrhage in atrial fibrillation patients during anticoagulation with warfarin or dabigatran: the RE-LY trial. Stroke. 2012;43(6):1511-1517. doi: 10.1161/STROKEAHA.112.650614.

24. Hankey GJ, Stevens SR, Piccini JP, et al. Intracranial hemorrhage among patients with atrial fibrillation anticoagulated with warfarin or rivaroxaban: the rivaroxaban once daily, oral, direct factor $\mathrm{Xa}$ inhibition compared with vitamin K antagonism for prevention of stroke and embolism trial in atrial fibrillation. Stroke. 2014;45(5):1304-1312. doi: 10.1161/STROKEAHA.113.004506.

25. Eikelboom JW, Wallentin L, Connolly SJ, et al. Risk of bleeding with 2 doses of dabigatran compared with warfarin in older and younger patients with atrial fibrillation : an analysis of the randomized evaluation of long-term an- 
ticoagulant therapy (RE-LY trial). Circulation. 2011;123(21):2363-2372. doi: 10.1161/CIRCULATIONAHA.110.004747.

26. El Ouali S, Barkun A, Martel M, Maggio D. Timing of rebleeding in highrisk peptic ulcer bleeding after successful hemostasis: a systematic review. Can J Gastroenterol Hepatol. 2014;28(10):543-548. doi: 0.1016/S00165085(14)60738-1.

27. Kimmel SE, French B, Kasner SE, et al. A pharmacogenetic versus a clinical algorithm for warfarin dosing. N Engl J Med. 2013;369(24):2283-2293. doi: 10.1056/NEJMoa1310669.

28. Healthcare Cost and Utilization Project (HCUP), Agency for Healthcare Research and Quality. HCUP Databases. https://www.hcup-us.ahrq.gov/ nisoverview.jsp. Accessed August 31, 2018.

29. Guerrouij M, Uppal CS, Alklabi A, Douketis JD. The clinical impact of bleeding during oral anticoagulant therapy: assessment of morbidity, mortality and post-bleed anticoagulant management. J Thromb Thrombolysis. 2011;31(4):419-423. doi: 10.1007/s11239-010-0536-7.

30. Fang MC, Go AS, Chang Y, et al. Death and disability from warfarin-associated intracranial and extracranial hemorrhages. Am J Med. 2007;120(8):700705. doi: 10.1016/j.amjmed.2006.07.034.

31. Guertin JR, Feeny D, Tarride JE. Age- and sex-specific Canadian utility norms, based on the 2013-2014 Canadian Community Health Survey. CMAJ. 2018;190(6):E155-E161. doi: 10.1503/cmaj.170317.

32. Gage BF, Cardinalli AB, Albers GW, Owens DK. Cost-effectiveness of warfarin and aspirin for prophylaxis of stroke in patients with nonvalvular atrial fibrillation. JAMA. 1995;274(23):1839-1845. doi: 10.1001/ jama.1995.03530230025025.

33. Fang MC, Go AS, Chang Y, et al. Long-term survival after ischemic stroke in patients with atrial fibrillation. Neurology. 2014;82(12):1033-1037. doi: 10.1212/WNL.0000000000000248.

34. Hong KS, Saver JL. Quantifying the value of stroke disability outcomes: WHO global burden of disease project disability weights for each level of the modified Rankin scale * Supplemental Mathematical Appendix. Stroke.
2009:40(12):3828-3833. doi: 10.1161/STROKEAHA.109.561365.

35. Jalal H, Dowd B, Sainfort F, Kuntz KM. Linear regression metamodeling as a tool to summarize and present simulation model results. Med Decis Mak. 2013;33(7):880-890. doi: 10.1177/0272989X13492014.

36. Staerk L, Lip GYH, Olesen JB, et al. Stroke and recurrent haemorrhage associated with antithrombotic treatment after gastrointestinal bleeding in patients with atrial fibrillation: nationwide cohort study. BMJ. 2015;351:h5876. doi: $10.1136 / \mathrm{bmj} . \mathrm{h} 5876$

37. Kopec JA, Finès P, Manuel DG, et al. Validation of population-based disease simulation models: a review of concepts and methods. BMC Public Health. 2010;10(1):710. doi: 10.1186/1471-2458-10-710.

38. Smith EE, Shobha N, Dai D, et al. Risk score for in-hospital ischemic stroke mortality derived and validated within the Get With The Guidelines-Stroke Program. Circulation. 2010;122(15):1496-1504. doi: 10.1161/CIRCULATIONAHA.109.932822

39. Smith EE, Shobha N, Dai D, et al. A risk score for in-hospital death in patients admitted with ischemic or hemorrhagic stroke. J Am Heart Assoc. 2013;2(1):e005207. doi: 10.1161/JAHA.112.005207.

40. Busl KM, Prabhakaran S. Predictors of mortality in nontraumatic subdural hematoma. J Neurosurg. 2013;119(5):1296-1301. doi: 10.3171/2013.4.JNS122236.

41. Murphy SL, Kochanek KD, Xu J, Heron M. Deaths: final data for 2012. Nat/ Vital Stat Rep. 2015;63(9):1-117. http://www.ncbi.nlm.nih.gov/pubmed/26759855. Accessed August 31, 2018.

42. Dachs RJ, Burton JH, Joslin J. A user's guide to the NINDS rt-PA stroke trial database. PLOS Med. 2008;5(5):e113. doi: 10.1371/journal.pmed.0050113.

43. Ashburner JM, Go AS, Reynolds K, et al. Comparison of frequency and outcome of major gastrointestinal hemorrhage in patients with atrial fibrillation on versus not receiving warfarin therapy (from the ATRIA and ATRIA-CVRN cohorts). Am J Cardiol. 2015;115(1):40-46. doi: 10.1016/j.amjcard.2014.10.006.

44. Weinstein MC, Siegel JE, Gold MR, Kamlet MS, Russell LB. Recommendations of the panel on cost-effectiveness in health and medicine. JAMA. 1996;276(15):1253-1258. doi: 10.1001/jama.1996.03540150055031. 